\title{
Effect of jet orientation on the mode of counter jet flow
}

\author{
by A.S. Ramamurthy
}

Associate Professor,

Sir George Williams University, Montreal and L. Robillard

Associate Professor,

Ecole Polytechnique. Montreal

\section{Introduction}

With the imposition of stricter pollution laws, the traditional methods of outlet design have undergone considerable revisions. Optimization of such structures are planned in terms of providing effective dispersion of the effluent in a short reach rather than in terms of accommodating the needs of dredging and navigation. A method of diluting the effluents in a flowing body of water consists of injecting the former as a counter jet (Figure 1).

This technical note deals with the effect of changing the jet orientation on the mode of the plane counter jet flow. In the stable mode, the jet flow does not oscillate. In the unstable mode, the jet oscillates and sweeps a wide area in the downstream section of the wake. Both visual observations and hot wire surveys have been made in the near wake of the jet to record and establish the frequency components of the velocity field. Recentiy, Robillard (1971) reported the existence of counter jet oscillations.

Besides jet orientation effects, the interference effects of the injection device on the mode of counter jet flow are also reported. The latter effect is termed as body interference effect.

\section{Experimental arrangement and procedure}

The experimental investigations were conducted in the test section of a water tunnel $\left(10^{\prime \prime} \times 72^{\prime \prime}\right)$ which was $96^{\prime \prime}$ long (Figure 2), The depth of flow was 65". A two-dimensional injecting device was designed to provide a plane jet flow which was $1 / 16^{\prime \prime}$ at the jet exit plane. The supply to the jet was from the sides of the water tunnel. The injecting device was a plate like structure which was $1^{\prime \prime}$ thick. For a given orientation $\alpha$ of the jet (Figure 1), the main flow velocity $U$ was fixed and the jet velocity $\mathrm{V}_{j}$ was increased in steps till the jet began to oscillate. This procedure was repeated for a range of $\alpha$ and $U$ to establish the upper range of the ratio $k=\left(V_{j} / U\right)$ which denoted the change from the stable mode to the unstable mode of the jet. To establish the lower range of the value of $k$ for fixed values of $\alpha$ and $U$, the jet velocity was increased to a very large value and decreased in steps till the jet switched from the unstable to the stable mode.

To study the body interference effects, the jet injecting device was surrounded by a plexiglass cylinder which was $7.75^{\prime \prime}$ in diameter. For these tests, $\alpha$ was fixed at zero degrees. Even during these tests, the upper and lower values of $k$ that denoted the changes in the jet mode were determined.

Visual observation of the flow was made possible by the use of dye which was injected in the supply line. The frequency of jet oscillation was low enough to be obtained directly with the help of a stop watch. However, this procedure was supplemented by means of the hot wire survey records in the wake region and in the vicinity of the jet exit plane. Figure 3 indicates a few hot film signal records related to the mode of the flow.

\section{Discussion of results}

For the discussion of results, the following parameters are defined. Accordingly,

Velocity ratio $k=\left(V_{j} / U\right)$

Kinematic jet momentum $J=V_{j}^{2} t$ 


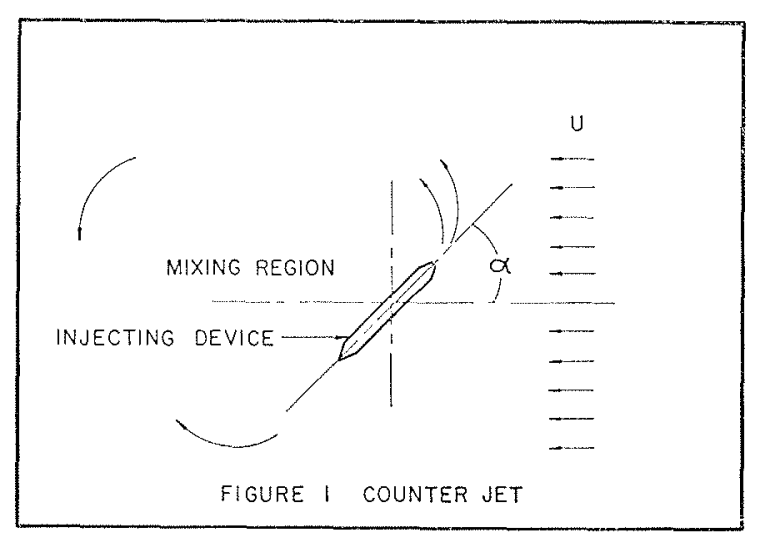

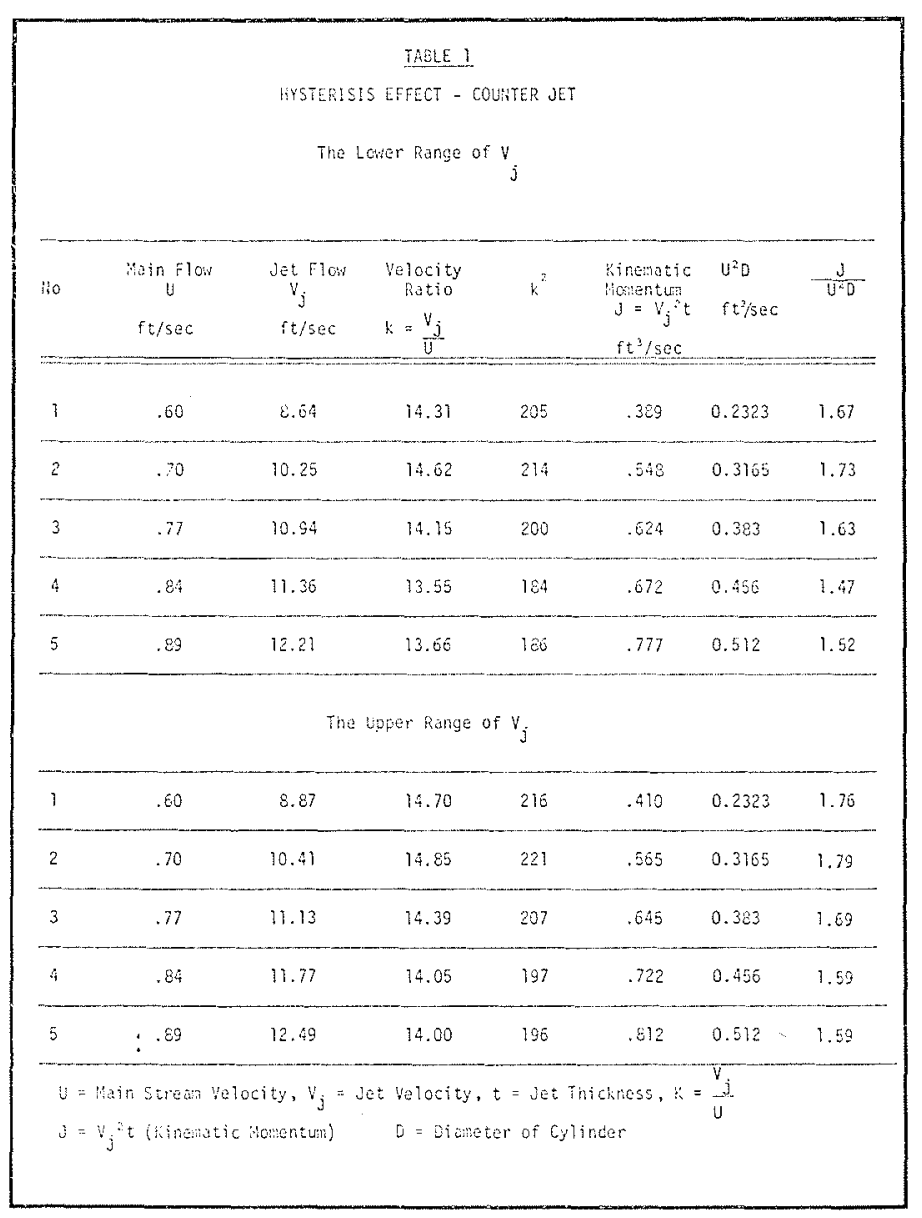
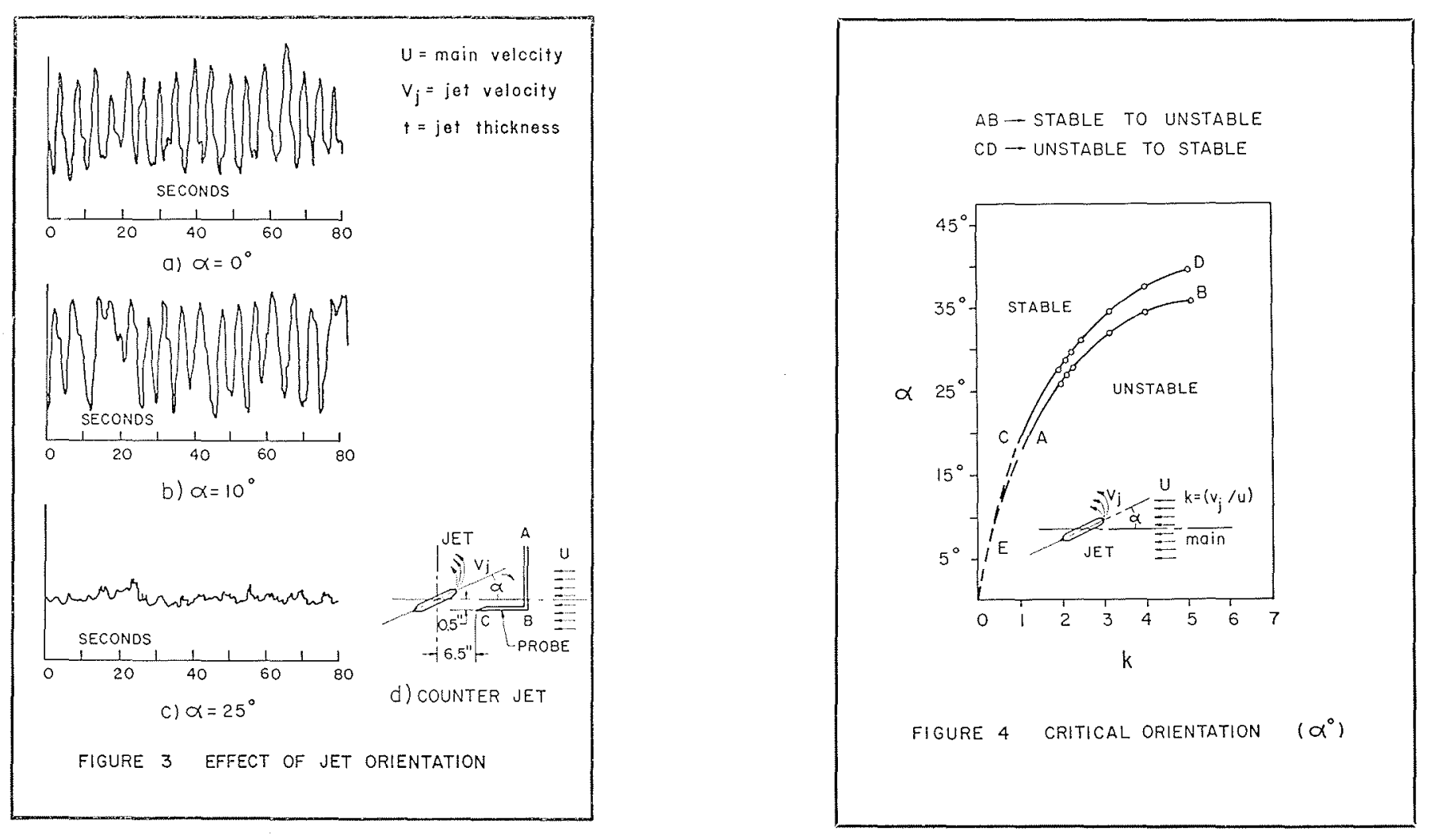
Momentum ratio $\lambda=\left(J / U^{2} l\right) \quad\left(k^{2} t / l\right)$ where,

$t$ is the jet thickness (initial)

$l$ is the length of injecting device (Figure 1)

\section{Rody Interference}

Table 1 indicates the results of boby interference tests in which the relatively large plexiglass cylinder influenced the jet mode $(\alpha=0)$ and is indicative of the hysterisis phenomena. Generally, the switch from one mode to the other was abrupt. In the stable mode, the dye injected into jet flow showed very little tendency to diffuse into the main stream, while in the unstable mode, the jet wake swept a large area and was indicative of large scale mixing.

\section{Effect of Jet Orientation}

For the studies related to jet orientation effets, the enveloping cylinder was removed. The results of tests related to the mode of jet flow as a function of jet orientation $\alpha$ and $k^{2}$ are plotted in Figure 4 . The solid lines indicate the transition from the stable to the unstable mode while the dashed lines indicate the transition from the unstable to the stable mode. Figure 3 displays the nature of the hotwire signal records registered at different orientations of the jet. While recording the signals, the upper cut off frequency of the filter was well above the frequency of jet oscillation and the frequency of vortex shedding of the injecting device itself. As one would expect, an inorease in the jet velocity relative to the main flow velocity promotes jet instability. In the unstable mode, the existence of low frequency (large scale) oscillations are discernible in the hot wire records (Figures $3 a$ and $3 b$ ). On the other hand, when the jet mode is stable, the hot wire record (Figure 3c) indicates that the low frequency components are absent. Clearly the small scale structure associated with the stable mode does not promote rapid mixing.

At large values of $\alpha$, the interference effects (Body interference) due to the inclined plate like structure may be considerable. The data reported is restricted to exploratory studies of the counter jet. Regarding the characteristics of hysterisis, these results only indicate general trends. For a different geometry of the injecting device the exact critical angles denoting the switching of jet modes may be different. For further studies, it is planned to vary the values of the initial jet thickness and the length $l$ (Figure 1) of the injecting device to generalize the results. Clearly, a longer plate length promotes stability to the jet, since the jet flow can reattach to a longer plate. This suggests the use of $\lambda=\left(\mathrm{J} / \mathrm{U}^{2} l\right)$ as the more appropriate parameter to denote the critical angle for the switching of the jet mode.

It is conceivable that the interference effects (Ramamurthy, 1971, Robillard, 1973) will have a strong influence on the mixing characteristics of the counterjet wake.

\section{Applications}

It is not unusual to have a finite orientation $(\alpha \not \neq 0)$ to the jet in a field structure to reduce the bed scour potential. Under such circumstances, effective mixing in a given stretch of the stream can be improved by limiting the orientation to angles which promote jet oscillations. The counter jet should be set in the unstable mode during the entire range of operation so that large scale mixing is present in the wake.

\section{References}

Robillard (L.) (1971). - “Mouvement périodique d'un jet bj dimensionnel plan dans un contre-courant et production de tourbillons a'ternés", Joumal de Mécanique, vol. 10, No. 1.

Ramamurthy (A.S.) and Robillard (L.) (1971). - "Phénomènes do transfert dans le cas d'un jet à contre-courant", Proceedings of the Intemational Association of Hydraulics Research, vol. 1, September 1971, pp. 167-174.

Robillard (L.) and Ramamurthy (A.S.) (1973). - "On the Characteristics of the Vortex Street Generated by a Counterjet", J. Fluids Engineering, T. A.S.M.E., vol. 96, No 1, March 1974 pp. 43-48. 